\title{
Carinal resections
}

\author{
Leonidas Tapias, Michael Lanuti
}

Division of Thoracic Surgery, Massachusetts General Hospital, Boston, MA, USA

Correspondence to: Michael Lanuti, MD. Division of Thoracic Surgery, Massachusetts General Hospital, 55 Fruit Street, Founders 7, Boston, MA 02114, USA. Email: mlanuti@mgh.harvard.edu.

Submitted Nov 20, 2017. Accepted for publication Dec 21, 2017.

doi: 10.21037/acs.2018.01.21

View this article at: http://dx.doi.org/10.21037/acs.2018.01.21

\section{Clinical vignette}

A 38-year-old non-smoking male presents with a 4-month history of cough and was referred to the Massachusetts General Hospital for management of a near obstructing mass at the left tracheobronchial angle. Bronchoscopy with biopsy revealed a mucoepidermoid carcinoma that extended from the distal trachea into the proximal left mainstem bronchus and spared the carina. He had no previous medical comorbidities. Chest computed tomography (CT) showed no significant extension beyond the airway and there was no associated lymphadenopathy. His pulmonary function was well preserved and he was brought forth for planned left carinal pneumonectomy. Of note, mediastinoscopy was performed at the time of surgery to mobilize the trachea and free the left recurrent laryngeal nerve from the tracheobronchial angle.

\section{Indications}

Carinal resections have been described as one of the most demanding operations in thoracic surgery. Carinal resections with or without lung resection are usually indicated for non-small cell lung cancer (NSCLC) involving the airway, low-grade malignant tumors or benign tumors (1). An extensive preoperative evaluation should be performed to assess the limits of resection, since lesions that require resecting more than $4 \mathrm{~cm}$ of distal trachea or more than $1.5 \mathrm{~cm}$ of proximal bronchus usually are not candidates.

\section{Preoperative evaluation}

All patients should undergo a physiologic evaluation, including pulmonary function testing and cardiac assessment. In patients with borderline pulmonary function, quantitative ventilation-perfusion scan and cardiopulmonary exercise testing should be considered. Cardiac evaluation should focus on identifying valvular disease or pulmonary hypertension.

The most common indication for carinal resection is non-small cell carcinoma. Complete evaluation should include CT and positron-emission tomography (PET) to identify metastatic disease precluding surgery, or advanced locoregional disease that may invoke neoadjuvant therapy. For central lesions magnetic resonance imaging (MRI) brain is recommended to rule out intracranial disease. Aggressive mediastinal staging with endobronchial ultrasound and/or mediastinoscopy is also recommended to identify patients with $\mathrm{N} 2 / \mathrm{N} 3$ disease. In cases where mediastinoscopy is needed, performing the procedure on the same day of planned resection will enhance proximal tracheal release and mobilization. If mediastinoscopy is performed weeks prior to the carinal resection, scarring of the pretracheal plane may limit the proximal release of the trachea translating to increased anastomotic tension. Flexible or rigid bronchoscopy is the ideal way to assess the extent of the tumor and obtain samples for histologic evaluation.

\section{Contraindications}

Insufficient cardiopulmonary reserve, pulmonary hypertension, and airway involvement greater than $4 \mathrm{~cm}$ are common contraindications. Tumor size is particularly crucial for left-sided resections. Mobility of the left hilum is limited by the aortic arch and resecting more than $4 \mathrm{~cm}$ of distal trachea poses a risk for tension and anastomotic dehiscence. A previous history of chest radiation should make the surgeon wary about potential complications, thus 


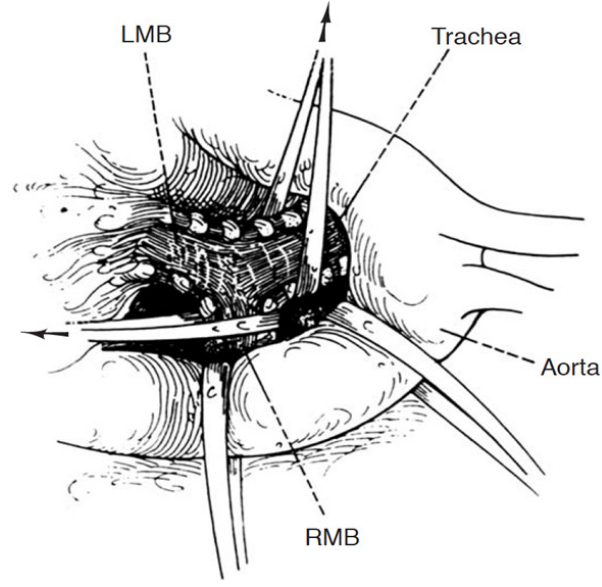

Figure 1 Left carinal exposure through left thoracotomy. Tapes around distal trachea and RMB (4). RMB, right main bronchus; LMB, left main bronchus.

prompting the strong recommendation to buttress the anastomosis (2).

\section{Operative technique}

\section{Anesthetic technique}

The anesthetic plan is crucial to the success of the operation. All efforts should be directed to extubating at the end of the procedure. Total intravenous anesthesia and preoperative epidural catheters contribute towards achieving this goal. Additionally, appropriate endotracheal tube selection is paramount. Flexible, extra-long single-lumen tubes allow for one-lung ventilation by advancement of the tube into the main-stem bronchus. Once resection is complete, crossfield ventilation is commenced using a sterile endotracheal tube connected to sterile extension tubing which is passed off to the anesthesiologist. It is important to protect the remaining lung by using small tidal volumes and avoiding continuous episodes of atelectasis. Ongoing communication between the surgeon and anesthesiologist guarantees adequate ventilation/oxygenation and allows precise placement of anastomotic sutures. Permissive hypercapnia is common during this phase of the operation.

\section{Rigid bronchoscopy}

Before committing to a carinal resection, flexible and often rigid bronchoscopy is recommended to estimate the extent of the disease, the amount of airway to be resected, and to assess the feasibility of reconstruction (3).

\section{Mediastinoscopy}

On the day of surgery, mediastinoscopy can be used to assess for extraluminal tumor extension. Additionally, dissection of the paratracheal planes make the subsequent mobilization of the trachea easier at the time of thoracotomy. Furthermore, sampling of mediastinal lymph nodes helps to identify patients who are not surgical candidates.

\section{Approach}

The right chest allows better access and visualization of the carina and both main-stem bronchi. This can be achieved via right posterior-lateral thoracotomy through the fourth or fifth intercostal space. The operative field should include the anterior neck, chest and ipsilateral arm in case additional release maneuvers are needed. The left chest poses difficult exposure due to the presence of the left-sided aortic arch. The aorta must be mobilized to gain access to the carina. Retraction tapes can be used to facilitate exposure (Figure 1). Video-assisted technology can help with the division of the left-sided pulmonary vessel to subsequently approach the airway reconstruction through a median sternotomy. Bilateral submammary transsternal thoracotomy (clamshell) and sternotomy are approaches to be considered but require proper patient selection.

\section{Release maneuvers}

One of the keys to success during carinal resection is to avoid tension at all costs. Creating an anastomosis under tension is a critical technical error that will lead to failure. The simplest maneuver is neck flexion allowing descent of the trachea into the mediastinum. Developing the anterior tracheal plane by mediastinoscopy or directly during thoracotomy provides mobility without interrupting the lateral blood supply.

Significant reduction in tension can be achieved by mobilizing the hilum, and should be done before starting any airway resection. Once the inferior pulmonary ligament is divided to the level of the inferior pulmonary vein, a U-shaped incision is made over the pericardium, below the hilum. Separating the inferior pulmonary vein from the inferior vena cava on the right side, allows the hilum to be shifted upwards. In situations where additional length is required, a complete hilar release can be performed. This 
is beneficial if re-implantation of the right main bronchus (RMB) or bronchus intermedius into the side of the trachea is planned.

Suprahyoid laryngeal release, helpful during tracheal resections, does not play a significant role during tracheobronchial resections. The distal mobility of the trachea achieved by this maneuver is not significant. Neck flexion should be performed at the time of anastomosis. A "guardian" stitch between the chin and chest reminds the patient to keep a mild degree of cervical flexion, avoiding neck extension and excessive tension in the early postoperative period. The stitch is usually removed on the seventh postoperative day and is well tolerated by the patient.

\section{Dissection}

As described by Dr. Grillo (2), the blood supply of the trachea is segmental, and interruption must be avoided. Lateral dissection should be within $2 \mathrm{~cm}$ of the area to be resected to prevent devascularization. Controversy exists about the extent of lymph node dissection. While some advocate for a more extensive lymphadenectomy, proper staging can be done by sampling and the use of frozen section, preventing excessive disruption of anastomotic blood supply and inadvertent recurrent laryngeal nerve injuries.

\section{Anastomosis}

To create an end-to-end anastomosis, we place traction sutures laterally, above and below the anastomosis. These 2-0 Vicryl (polyglactin; Ethicon, Inc, Somerville, New Jersey, USA) sutures are fastened across the anastomosis to reduce tension, once the airway is reapproximated. Simple interrupted sutures of 4-0 Vicryl are placed in a concentric fashion $3-4 \mathrm{~mm}$ apart and $3-4 \mathrm{~mm}$ from the cut edge with the knots lying outside of the airway. Suture placement can be slightly modified to correct size discrepancy between both ends. We cannot stress enough the importance of handling the bronchial mucosa gingerly to avoid any trauma. Once the sutures are placed and tagged, the endotracheal tube is advanced under direct vision beyond the anastomosis. Traction sutures are secured, all anastomotic sutures are tied and an air leak test is performed. Caution should be exercised when placing repair sutures and most of the time, reconstructing the anastomosis will be required.
End-to-side anastomosis follows the same principles. However, two additional technical points must be considered. First, the side opening must be at least $1 \mathrm{~cm}$ away from the end-to-end anastomosis to avoid devascularization and necrosis of the intervening airway segment, and second, it must be created entirely within the cartilaginous portion of the airway. Avoiding the membranous wall provides additional rigidity at the level of the anastomosis.

One should always aim for a tension-free anastomosis and clear surgical margins. Unfortunately, in cases where a safe reconstruction might not be achieved, a tensionfree reapproximation takes precedence. The outcomes of a dehisced anastomosis are catastrophic and unbearable for the patient and the surgeon (5). Once all suture lines are tested, we favor wrapping each anastomosis with viable tissue, to separate suture lines from more delicate structures such as pulmonary vessels. We favor the use of pleura, pedicled pericardial fat pad, omentum or intercostal muscle flaps. The caveat with utilization of an intercostal muscle flap, due to the possible presence of periosteum, is that when circumferentially wrapped, it can produce bony encasement of the airway with late stenosis.

\section{Resection and reconstruction}

The planned resection and reconstruction is based on the location of the tumor. It can consist of a carinal resection with right pneumonectomy or right upper lobectomy, left pneumonectomy or pure carinal resection (6).

In cases where a limited resection of the carina is needed, the right and left main-stem bronchi can be reapproximated to create a "neocarina" which is anastomosed to the distal trachea. The main limitation in this reconstruction is the restriction of mobilizing the neocarina cephalad due to the aortic arch. In this case, mobilization is based on releasing maneuvers of the trachea (Figure 2). However, if the tracheal segment needing resection is less than $4 \mathrm{~cm}$, an end-to-end anastomosis between the trachea and the left main bronchus (LMB) with reimplantation of the RMB into the side of the trachea is recommended (Figure 3).

When more than $4 \mathrm{~cm}$ of distal trachea is necessary, an end-to-end anastomosis between the trachea and the $\mathrm{RMB}$ with reimplantation of the LMB into the side of the bronchus intermedius should be used (Figure 4).

In situations where tumor compromises either of the main bronchi, the lung resection is performed (right upper lobectomy, right or left pneumonectomy) and an end-to- 


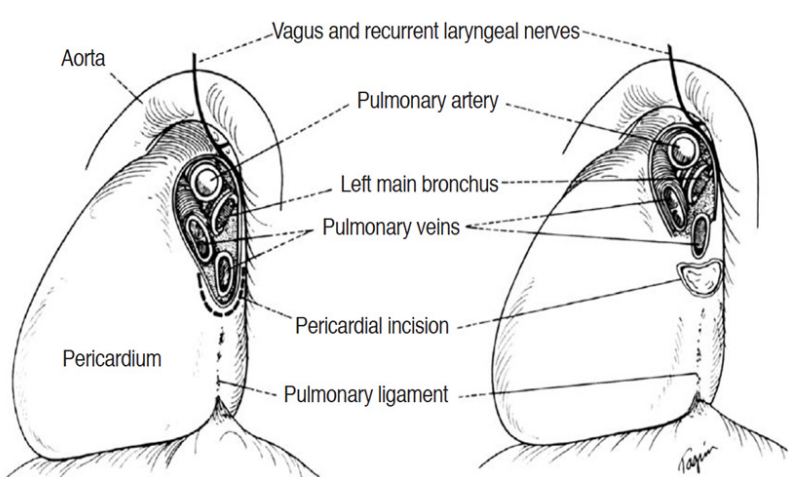

Figure 2 Left-sided intrapericardial hilar release technique demonstrating the U-shaped pericardial incision to facilitate the creation of a tension-free anastomosis (4).

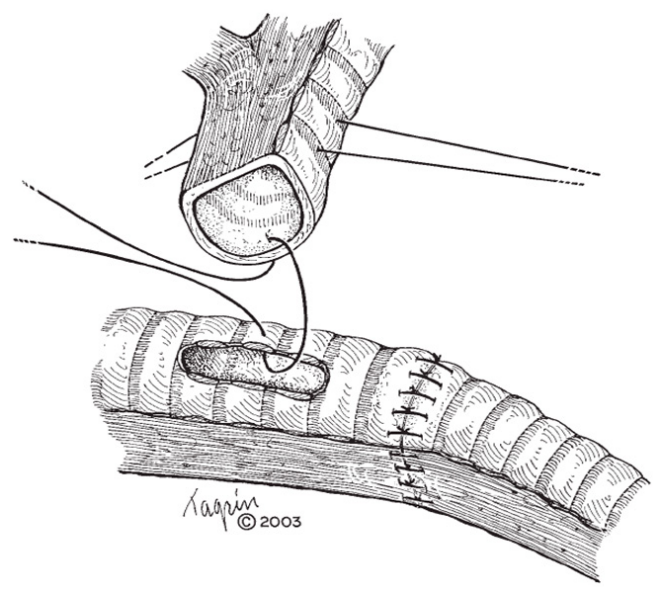

Figure 3 Creation of an end-to-end anastomosis between the trachea and the left main bronchus with reimplantation of the right main bronchus. Created opening is entirely in the cartilaginous wall to provide rigidity (7).

end anastomosis is created between the trachea and the remaining bronchus (Figure 5).

\section{Postoperative management}

It is imperative to extubate these patients upon completion of the procedure to protect the newly created anastomosis. Flexible bronchoscopy is performed to assess the anastomosis and clean the airway of blood and secretions. On postoperative day $5-7$, flexible bronchoscopy should be repeated to evaluate the anastomosis and assess the release of the guardian stitch. Strict fluid management to protect the contralateral lung is recommended after pulmonary

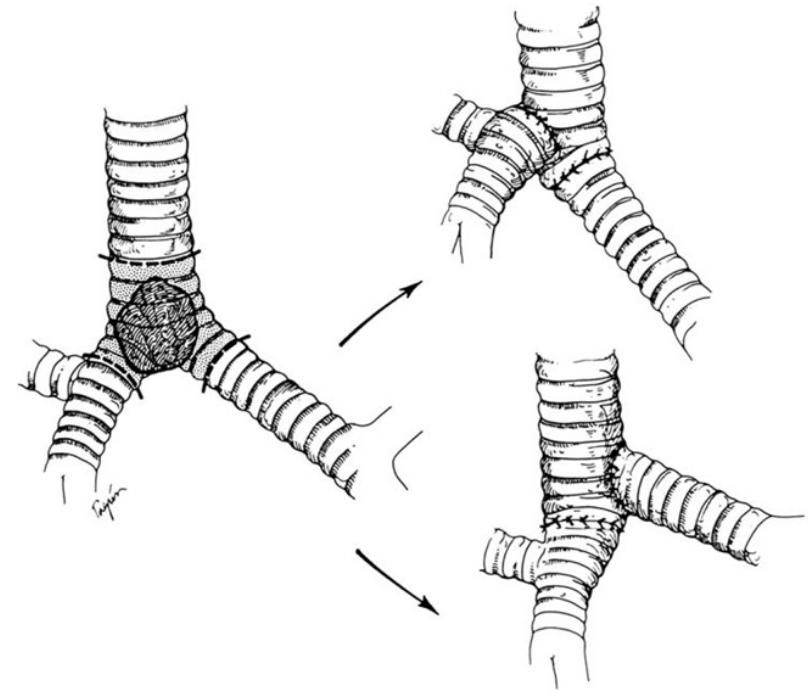

Figure 4 Carinal reconstruction with extensive airway resection. The trachea can be anastomosed end to end with either the right or left main-stem bronchus, with the contralateral bronchus reimplanted into the side of the trachea (8).

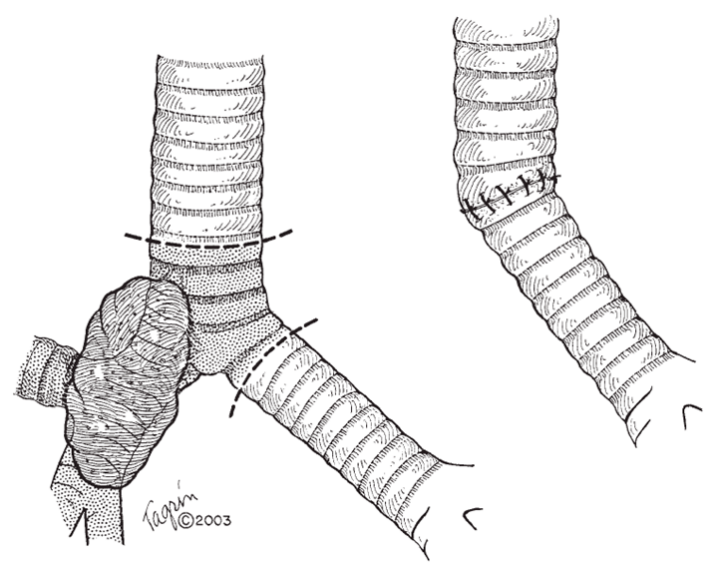

Figure 5 Trachea and contralateral bronchus are anastomosed in an end-to-end fashion (8).

resection.

\section{Comments}

Despite being a technically challenging procedure, outcomes are directly related to the volume of the center. Postoperative mortality ranges between $3-12 \%$ in high volume centers (5). The primary predictor of mortality is related to the baseline disease process. In patients 
with malignant disease, the 5 -year overall survival in the absence of $\mathrm{N} 2$ disease ranges from $38 \%$ to $53 \%(1,5,9)$. Perioperative mortality is profoundly influenced by the presence of non-cardiogenic pulmonary edema and pneumonia. The need for mechanical ventilation in this setting can potentiate anastomotic complications and leads to significant decrease in overall survival. Judicious patient selection and application of correct surgical principles make this operation an excellent option for patients with tumors extending to the airway.

\section{Acknowledgements}

None.

\section{Footnote}

Conflicts of Interest: The authors have no conflicts of interest to declare.

\section{References}

1. Mitchell JD, Mathisen DJ, Wright CD, et al. Resection for bronchogenic carcinoma involving the carina: long-term results and effect of nodal status on outcome. J Thorac Cardiovasc Surg 2001;121:465-71.

2. Grillo HC. Carinal reconstruction. Ann Thorac Surg 1982;34:356-73.

3. Lanuti M, Mathisen DJ. Carinal resection. Thorac Surg Clin 2004;14:199-209.

4. Mathisen DJ, Grillo HC, Gaissert HA. Carinal resection. In: Pearson FG, Cooper JD, Deslauriers J, et al, editors. Thoracic surgery. 2nd edition. New York: Churchill Livingstone, 2002;415-25.

5. Tapias LF, Ott HC, Mathisen DJ. Complications Following Carinal Resections and Sleeve Resections. Thorac Surg Clin 2015;25:435-47.

6. Grillo HC. Carcinoma of the lung: what can be done if the carina is involved? Am J Surg 1982;143:694-5.

7. Grillo HC. Surgery of the Trachea and Bronchi. Houston: BC Decker, 2004.

8. Mitchell JD, Mathisen DJ, Wright CD, et al. Clinical experience with carinal resection. J Thorac Cardiovasc Surg 1999;117:39-52.

9. Weder W, Inci I. Carinal resection and sleeve pneumonectomy. J Thorac Dis 2016;8:S882-8.
Cite this article as: Tapias L, Lanuti M. Carinal resections. Ann Cardiothorac Surg 2018;7(2):309-313. doi: 10.21037/ acs.2018.01.21 